H. KOMIYA

KODAI MATH. J.

11 (1988), 5-7

\title{
ELEMENTARY PROOF FOR SION'S MINIMAX THEOREM
}

\author{
By Hidetoshi KomiYa
}

\section{Introduction.}

There are several celebrated generalizations of von Neumann's minimax theorem, one of which is the following by Sion [2]:

Let $X$ be a compact convex subset of a linear topological space and $Y$ a convex subset of a linear topological space. Let $f$ be a real-valued function on $X \times Y$ such that

(i) $f(x, \cdot)$ is upper semicontinuous and quasi-concave on $Y$ for each $x \in X$;

(ii) $f(\cdot, y)$ is lower semicontinuous and quasi-convex on $X$ for each $y \in Y$. Then

$$
\min _{x \in X} \sup _{y \in Y} f(x, y)=\sup _{y \in Y} \min _{x \in X} f(x, y) .
$$

Sion proved the theorem using Knaster-Kuratowski-Mazurkiewicz theorem (or shortly KKM theorem). Alternative proofs for the theorem were proposed by several authors. For example, Fan [1] deduced the theorem from his theorem concerning sets with convex sections. Takahashi [3] derived the theorem from Fan-Browder fixed point theorem for multi-valued mappings. However their proofs depend on topological tools such as Brouwer fixed point theorem or KKM theorem. The purpose of this note is to present an elementary proof for Sion's minimax theorem.

\section{Proof for the theorem.}

The method of our proof is inspired by the proof of [4, Theorem 2].

LEMMA 1. Under the same assumptions of Sion's theorem, for any $y_{1}$ and $y_{2} \in Y$ and any real number $\alpha$ with $\alpha<\min _{x \in X} \max \left(f\left(x, y_{1}\right), f\left(x, y_{2}\right)\right)$, there is $y_{0} \in Y$ with $\alpha<\min _{x \in X} f\left(x, y_{0}\right)$.

Proof. Deny the conclusion and suppose that $\alpha \geqq \min _{x \in X} f(x, y)$ for all $y \in Y$. Choose $\beta$ with

$$
\alpha<\beta<\min _{x \in X} \max \left(f\left(x, y_{1}\right), f\left(x, y_{2}\right)\right) .
$$

Received June 19, 1987 
Denote by $\left[y_{1}, y_{2}\right]$ the line segment joining $y_{1}$ and $y_{2}$, and for each $z \in\left[y_{1}, y_{2}\right]$, define

$$
C z=\{x \in X: f(x, z) \leqq \alpha\}, \quad C^{\prime} z=\{x \in X: f(x, z) \leqq \beta\},
$$

$A=C^{\prime} y_{1}$ and $B=C^{\prime} y_{2}$. Then $C z, C^{\prime} z, A$ and $B$ are all nonempty, closed by the lower semicontinuity of $f(\cdot, z)$, and $A \cap B=\phi$. By the quasi-concavity of $f(x, \cdot)$,

$$
f(x, z) \geqq \min \left(f\left(x, y_{1}\right), f\left(x, y_{2}\right)\right)
$$

for $x \in X$ and $z \in\left[y_{1}, y_{2}\right]$. Hence we have $C^{\prime} z \subset A \cup B$. Since $C^{\prime} z$ is convex by the quasi-convexity of $f(\cdot, z)$, we have $C^{\prime} z$ is connected, and hence

Define

$$
C z \subset C^{\prime} z \subset A \text { or } C z \subset C^{\prime} z \subset B \text {. }
$$

$$
I=\left\{z \in\left[y_{1}, y_{2}\right]: C z \subset A\right\} \text { and } J=\left\{z \in\left[y_{1}, y_{2}\right]: C z \subset B\right\},
$$

then $I$ and $J$ are nonempty, $I \cap J=\phi$ and $I \cup J=\left[y_{1}, y_{2}\right]$. Let $\left\{z_{n}\right\}$ be a sequence in $I$ with $\lim z_{n}=z \in\left[y_{1}, y_{2}\right]$. Let $x$ be any point of $C z$. Then we have $f(x, z)$ $<\beta$. By the upper semicontinuity of $f(x, \cdot)$, we have $\overline{\lim } f\left(x, z_{n}\right)<\beta$. Hence there is an integer $m$ with $f\left(x, z_{m}\right)<\beta$, that is, $x \in C^{\prime} z_{m}$. We have $C^{\prime} z_{m} \subset A$ since $C z_{m} \subset A$, and hence $x \in A$. That is, $z \in I$ and $I$ is closed in $\left[y_{1}, y_{2}\right]$. Similarly we can show that $J$ is closed. The closedness of both $I$ and $J$ contradicts the connectedness of $\left[y_{1}, y_{2}\right]$. This completes the proof.

LEMMA 2. Under the same assumptions of Sion's theorem, for any finite $y_{1}, \cdots, y_{n} \in Y$ and any real number $\alpha$ with $\alpha<\min _{x \in X} \max _{1 \leqq \imath \leqq n} f\left(x, y_{\imath}\right)$, there is $y_{0} \in Y$ with $\alpha<\min _{x \in X} f\left(x, y_{0}\right)$.

Proof. We prove this by induction on $n$. Lemma 2 is trivial for $n=1$. Let $X^{\prime}$ be the compact convex set $\left\{x \in X: f\left(x, y_{n}\right) \leqq \alpha\right\}$. We may assume that $X^{\prime}$ is nonempty, otherwise we take $y_{0}=y_{n}$. Since $\alpha<\min _{x \in X} \max _{1 \leqq \imath n} f\left(x, y_{\imath}\right)$, we have

$$
\alpha<\min _{x \in X^{\prime}} \max _{1 \leqq \imath \leqq n-1} f\left(x, y_{\imath}\right) .
$$

We apply our induction-assumption to the restriction of $f$ to $X^{\prime} \times Y$. Then there is $y_{0}^{\prime}$ with $\alpha<\min _{x \in X^{\prime}} f\left(x, y_{0}^{\prime}\right)$. Hence we have $\alpha<\min _{x \in X} \max \left(f\left(x, y_{0}^{\prime}\right), f\left(x, y_{n}\right)\right)$, and there is $y_{0} \in Y$ with $\alpha<\min _{x \in X} f\left(x, y_{0}\right)$ by Lemma 1 .

Proof of the theorem. It is obvious that $\sup _{y \in X} \min _{x \in X} f(x, y) \leqq \min _{x \in X} \sup _{y \in Y} f(x, y)$. Hence we show the reverse inequality. Let $\alpha$ be any real number with $\alpha<$ $\min _{x \in X} \sup _{y \in Y} f(x, y)$ and let $X_{y}$ be the compact set $\{x \in X: f(x, y) \leqq \alpha\}$ for each $y \in Y$. Then $\bigcap_{y \in Y} X_{y}$ is empty, and hence there are finite $y_{1}, \cdots, y_{n} \in Y$ such 
that $\bigcap_{\imath=1}^{n} X_{y_{\imath}}$ is empty, that is, $\alpha<\min _{x \in X} \max _{1 \leq \imath \leq n} f\left(x, y_{\imath}\right)$. By Lemma 2, there is $y_{0} \in Y$ with $\alpha<\min _{x \in X} f\left(x, y_{0}\right)$, and hence $\alpha<\sup _{y \in X} \min _{x \in X} f(x, y)$. Therefore we have

$$
\min _{x \in X} \sup _{y \in Y} f(x, y) \leqq \sup _{y \in Y} \min _{x \in X} f(x, y) .
$$

Acknowledgement. The author would like to express his thanks to Professor W. Takahashi for his valuable advice.

\section{REFERENCES}

[1] FAN, K., Sur un théorème minimax, C. R. Acad. Sci. Paris 259 (1964), 3925-3928.

[2] Sion, M., On general minimax theorems, Pacific J. Math. 8 (1958), 171-176.

[3] TAKAhashi, W., Nonlinear variational inequalities and fixed point theorems, J. Math. Soc. Japan 28 (1976), 168-181.

[4] Terkelsen, F., Some minimax theorems, Math. Scand. 31 (1972), 405-413.

College of Commerce

NiHON UNIVERSITY

Kinuta, Setagaya-Ku

TOKYO 157, JAPAN 